\title{
BMJ Open Health and social consequences of an alcohol-related admission to critical care: a qualitative study
}

\author{
Joanne McPeake, ${ }^{1,2}$ Ewan Forrest, ${ }^{1}$ Tara Quasim, ${ }^{1,2}$ John Kinsella, ${ }^{1,2}$ Anna O'Neill ${ }^{2}$
}

To cite: McPeake J,

Forrest E, Quasim T, et al. Health and social

consequences of an alcoholrelated admission to critical care: a qualitative study. BMJ Open 2016;6:e009944. doi:10.1136/bmjopen-2015009944

- Prepublication history and additional material is available. To view please visit the journal (http://dx.doi.org/ 10.1136/bmjopen-2015009944).

Received 9 September 2015 Revised 22 December 2015 Accepted 6 January 2016
CrossMark

\footnotetext{
${ }^{1}$ Glasgow Royal Infirmary, Glasgow, UK

University of Glasgow, School of Medicine, Glasgow Royal Infirmary, Glasgow, UK

Correspondence to Joanne McPeake; joanne. mcpeake@glasgow.ac.uk
}

\section{ABSTRACT}

Objective: To examine the impact of critical care on future alcohol-related behaviour. Further, it aimed to explore patterns of recovery for patients with and without alcohol use disorders beyond the hospital environment.

Design: In-depth, semistructured interviews with participants (patients) 3-7 months post intensive care discharge.

Setting: The setting for this study was a 20-bedded mixed intensive care unit (ICU), in a large teaching hospital in Scotland. On admission, patients were allocated to one of the three alcohol groups: low risk, harmful/hazardous and alcohol dependency.

Participants: 21 participants who received mechanical ventilation for greater than 3 days were interviewed between March 2013 and June 2014.

Interventions: None.

Measurements and main results: Four themes which impacted on recovery from ICU were identified in this patient group: psychological resilience, support for activities of daily living, social support and cohesion and the impact of alcohol use disorders on recovery. Participants also discussed the importance of personalised goal setting and appropriate and timely rehabilitation for alcohol-related behaviours during the critical care recovery period.

Conclusions: There is a significant interplay between alcohol misuse and recovery from critical illness. This study has demonstrated that at present, there is a haphazard approach to rehabilitation for patients after ICU. A more targeted rehabilitation pathway for patients leaving critical care, with specific emphasis on alcohol misuse if appropriate, requires to be generated.

\section{INTRODUCTION}

Patients recovering from critical illness can have persistent physical, social and psychological problems in the months and years following discharge. ${ }^{1-3}$ Commonly reported problems include poor mobility, chronic pain, muscle wasting, poor sleep, depression and post-traumatic stress disorder (PTSD). ${ }^{2} \quad 4-7$ However, less is known about the impact of health-related behaviours following intensive care discharge.

\section{Strengths and limitations of this study}

- This is the first study, as far as we can establish, which has explored recovery in critically ill patients with alcohol use disorders (AUDs) beyond the hospital setting.

- This study has used a rigorous approach to qualitative analysis including peer review, explicit use of an analytical framework and the use of an audit trail.

- A limitation of this study is that it took place in a single centre, in an area of high deprivation.

Alcohol-related admissions to intensive care are increasing. ${ }^{8-14}$ Emerging evidence suggests that alcohol-related admissions represent a significant proportion of all critical care admissions, with up to $35 \%$ being directly or indirectly related to alcohol. ${ }^{13} 14$ There is also evidence demonstrating poor longer term outcomes for those patients admitted with a background of alcohol dependency, with three recent studies demonstrating poorer outcomes in this patient cohort up to 3 years post intensive care unit (ICU) discharge. ${ }^{13-15}$ However, there is uncertainty about the optimum rehabilitation approach for this group of patients and the impact which alcohol use disorders (AUDs) have on recovery. ${ }^{16}$ Further, the impact of AUDs on mortality in ICU is well understood. Long-term morbidity in this group, however, is understudied.

At present, there appears to be only one study which has explored this area. Clark et $a l^{17}$ conducted a qualitative study in the ICU environment to identify themes surrounding the decision to change drinking behaviours. Their aim was to specifically tailor brief interventions for AUDs in medical ICU survivors. ${ }^{17}$ This research identified five broad themes that facilitated the decision to cut down or stop drinking in survivors of ICU: empathy of the inpatient healthcare environment, recognition of 
accumulating problems, religion, pressure from others to stop drinking and the impact of trigger events. However, Clark et $a l^{17}$ interviewed patients within the ICU context and not during the recovery phase. As a result, these decisions and intentions may have changed over time, especially after discharge from the hospital setting.

The present study aimed to examine and understand the impact of critical illness on future behaviour with regard to alcohol intake and explore patterns of physical and psychological recovery for patients with and without AUDs beyond the hospital environment. It also aimed to understand the impact of critical illness on alcoholrelated behaviours after hospital discharge.

\section{MATERIALS AND METHODS}

This study was part of a larger, mixed-methods study exploring the health and social consequences of admission to ICU.

In-depth, semistructured interviews were conducted to address the research objectives. This method of data collection was chosen as this area of research is complex and, in some cases, deeply sensitive. Further, it was important to understand contextual accounts from participants about their recovery from ICU and behaviours regarding alcohol use. These contextual accounts would have been difficult to capture by any other research method. All interviews were audio recorded and then transcribed verbatim.

Interpretative phenomenological analysis (IPA) was chosen as an analytical framework for this study. ${ }^{18}$ IPA is concerned with understanding, exploring and interpreting the personal, lived experience of a participant, which was a key aim of this study. The six steps proposed by Smith et $a l^{18}$ were used for analysis: reading and re-reading of transcripts; initial coding; development of emergent themes; searching for connections across emergent themes; moving to the next case and looking for patterns. From this process, the creation of themes and superordinate themes took place.

All interviews were undertaken by the first author (JMcP) between February 2013 and February 2014. $\mathrm{JMcP}$ is an ICU nurse. An attempt to remove all previous knowledge and experience was made during the interview and analysis process. An audit trail was kept throughout the interview process. These notes, as well as all transcribed interviews, were given to external peer reviewers, who were experts in qualitative research as well as critical care practitioners, to review. This process aimed to reduce any bias and ensure all interpretations were based on data gathered rather than experience.

The study took place in one adult critical care unit in Glasgow. The hospital is a University Teaching Hospital within NHS Greater Glasgow and Clyde, Scotland. It is situated in an area of high socioeconomic deprivation, with $42 \%$ of the most deprived geographical areas in Scotland residing in this catchment area. ${ }^{19}$ In addition, this hospital is a tertiary referral centre for pancreatic care, burn care, oesophageal surgery and some orthopaedic interventions.

On admission to ICU, patients were prospectively assigned to one of the three groups on the basis of previous medical notes and information from the family and patient. The study groups used were based on the WHO's International Classification of Diseases, 10th edition (ICD-10) definition for AUDs: ${ }^{20}$ low risk, harmful/hazardous and alcohol dependency (box 1). Information on alcohol history was obtained by the admitting member of medical staff. No validated tool for alcohol misuse was used in the critical care unit in this study. Research demonstrates that the type of approach used in this study is typical of many ICUs in the UK. ${ }^{21}$

Participants were purposively recruited 3-7 months after ICU discharge. This time frame was chosen to allow the participants enough time to readjust to being home and to allow the research team to look at recovery and decision-making regarding alcohol use as it was actively happening. This time frame was also chosen to help reduce memory bias or recall bias with regard to the ICU experience.

Box 1 Definitions of alcohol use disorders (Adapted from $\mathrm{WHO}^{20}$ )

No risk/Low risk

- Individuals who;

- Consume no alcohol;

- Have experienced no or minimal harm as a result of alcoho use.

\section{Harmful use}

- Alcohol is responsible for or has substantially contributed to physical or psychological harm, including impaired judgement or dysfunctional behaviour;

- The nature of harm is clearly identifiable (ie, falls/absence from work);

- The pattern has persisted for at least 1 month previous to admission or has occurred repeatedly within a 12-month period.

Alcohol dependence (should be made if three or more of the following are present)

- A strong desire or sense of compulsion to take alcohol;

- Difficulty in controlling drinking in terms of onset, termination or level of use;

- A physiological withdrawal state is present when drinking has ceased or been reduced;

- Drinking to relieve or avoid withdrawal symptoms;

- Evidence of tolerance, such that increased doses of alcohol are required to achieve effects originally produced by lower amounts (examples are when individuals take daily doses sufficient to incapacitate or severely hurt non-tolerant users);

- Preoccupation with alcohol use to the detriment of other interests (eg, social or occupational);

- Persistent alcohol use despite awareness of harmful consequences, such as physical harm (liver impairment), depressive mood states consequent to periods of heavy drinking or alcohol-related impairment of cognitive function. 
The inclusion criteria for the semistructured interviews were as follows: patients who had been admitted to the ICU and ventilated for greater than $72 \mathrm{~h}$, patients who were older than 18 years of age at the time of ICU admission, patients who were younger than 75 years of age at the time of ICU admission, patients who were able to give full consent at the time of interview and patients who could speak English fluently (no requirement for translator). Patients who had ongoing mental health issues (such as alcohol-related brain damage) were excluded from the study. The term level three patient refers to the UK Intensive Care Society's definition of ICU patients. Level three patients require multiple organ support or invasive respiratory support only. ${ }^{22}$ Within the UK context, level three patients require to be supported within a critical care environment.

\section{Findings}

The present study aimed to examine and understand the impact of critical illness on future behaviour with regard to alcohol intake and explore patterns of recovery for patients with and without AUDs beyond the hospital

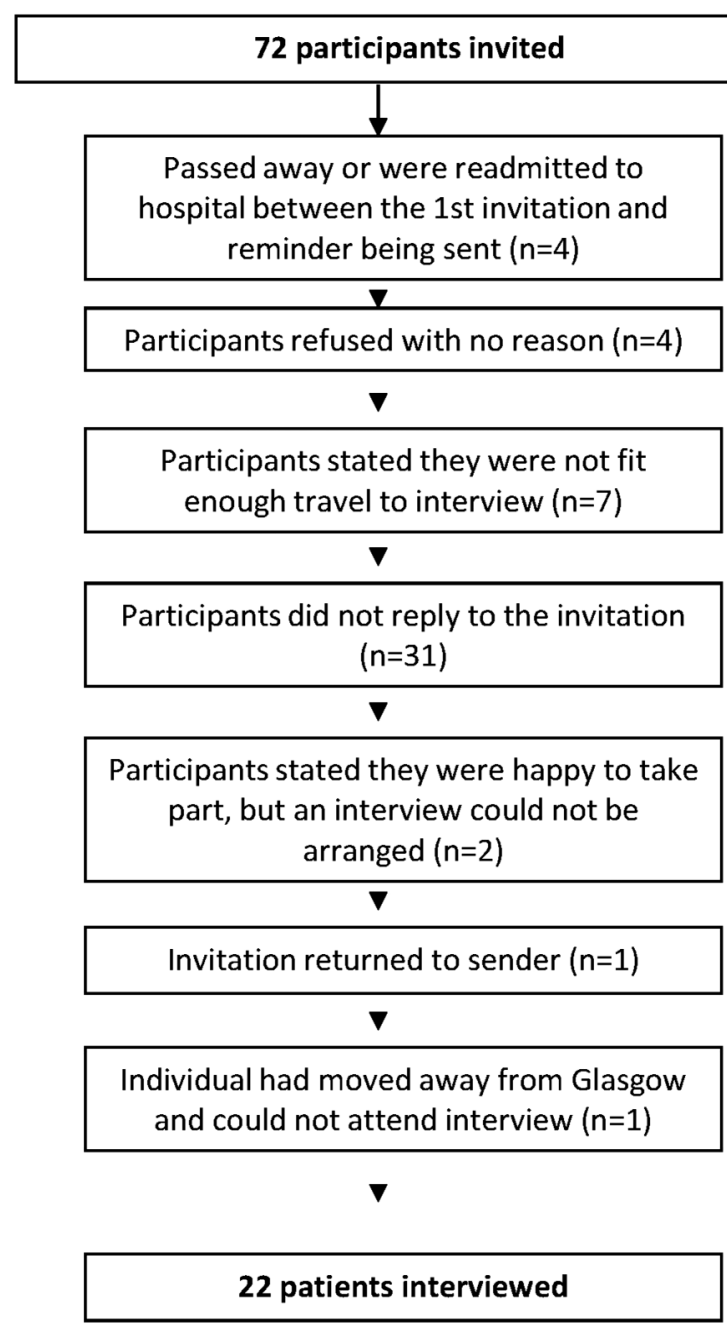

Figure 1 Responses to invitations sent for in-depth, semistructured interviews. environment. It also aimed to understand the impact of critical illness on alcohol-related behaviours after hospital discharge. The interview schedule is shown in online supplementary file 1 . Seventy-two participants were invited to take part in this study via a letter of invitation to their home address after hospital discharge. The responses to invitations sent are shown in figure 1 .

One pilot interview was undertaken to test the interview schedule and approach to the interview by the researcher. The pilot interview was not included in this final analysis. Data saturation was met at 19 interviews. Two further interviews were completed to ensure no new themes were generated. An overview of the interviews and the baseline characteristics of interview participants are presented in tables 1 and 2.

Nineteen interviews were undertaken in the family room adjacent to the ICU; one interview was undertaken in a sheltered housing complex and the final interview took place within the university. During several interviews, family members and/or next of kin were present.

Four themes were generated from this study (table 3). When undertaking and analysing the findings from the interviews, it became apparent that participants from the three study groups had similar problems and experiences during their recovery from ICU. However, for those admitted to the ICU with an AUD, there was a significant interplay between alcohol and their recovery from critical illness. Therefore, the first three themes (impact on activities of daily living (ADLs), impact of psychological resilience on recovery and social support and cohesion), with their corresponding superordinate themes, represent the entire cohort interviewed. Specific differences between the three study groups are highlighted. The final theme relates to those patients interviewed who were admitted to the ICU with an AUD.

\section{Impact on ADLs}

Descriptions of poor mobility were typically the starting point for participants discussing their recovery. Participants discussed how poor mobility often had a negative impact on their recovery:

Well...actually...I tried to get up and down stairs...with the two sticks, and I found it very, very hard. I was terrified actually. I was actually shaking.

It was also clear from the interviews that participants suffered many complex psychological problems after discharge from critical care. These problems appeared to be similar across the three study groups. Problems included low mood and increased anxiety about adapting to changes in health and adjusting to life after critical care:

I've had panic attacks and I've had to run back in the house just to be safe and sound.

Participants also described changes in sleeping patterns after discharge from intensive care and the effect 
Table 1 Overview of participants involved in the in-depth, semistructured interviews

\begin{tabular}{|c|c|c|c|c|c|}
\hline Age & Gender & Reason for ICU admission & Alcohol group & APACHE II & ICU LOS \\
\hline 73 & $\mathrm{~F}$ & Pneumonia & Low risk & 14 & 4 \\
\hline 70 & $M$ & Alcohol-related pancreatitis & Harmful/hazardous & 17 & $3^{*}$ \\
\hline 34 & $\mathrm{~F}$ & Alcohol-related pancreatitis & Alcohol dependence & 14 & 12 \\
\hline 52 & M & Bowel obstruction & Low risk & 22 & 5 \\
\hline 23 & $\mathrm{~F}$ & Out-of-hospital cardiac arrest & Low risk & 29 & 3 \\
\hline 57 & M & Pneumonia & Alcohol dependence & 19 & 11 \\
\hline 57 & M & Gl tract sepsis (Salmonella) & Alcohol dependence & 31 & 58 \\
\hline 31 & M & Serious assault & Harmful/hazardous & 20 & 26 \\
\hline 68 & $M$ & Necrotising fasciitis & Low risk & 30 & 6 \\
\hline 37 & $\mathrm{~F}$ & Urinary tract infection/sepsis & Alcohol dependence & 29 & 5 \\
\hline 63 & $\mathrm{~F}$ & Pneumonia & Low risk & 16 & 8 \\
\hline 54 & M & Pneumonia & Harmful/hazardous & 32 & 60 \\
\hline 52 & $\mathrm{~F}$ & Pneumonia & Low risk & 26 & 37 \\
\hline 63 & $\mathrm{~F}$ & Status epilepticus & Low risk & 19 & 3 \\
\hline 60 & M & Variceal bleed & Alcohol dependence & 23 & 3 \\
\hline 22 & M & Burn injury & Harmful/hazardous & 14 & 6 \\
\hline 59 & M & Accidental overdose & Harmful/hazardous & 30 & 4 \\
\hline 38 & M & Perforated DU & Alcohol dependence & 9 & 9 \\
\hline 50 & M & Metabolic disturbance & Alcohol dependence & 32 & 4 \\
\hline 40 & M & ARDS & Alcohol dependence & 33 & 9 \\
\hline 60 & M & Pneumonia & Alcohol dependence & 14 & 11 \\
\hline 39 & M & Out-of-hospital cardiac arrest & Harmful/hazardous & 23 & 4 \\
\hline
\end{tabular}

APACHE, Acute Physiology and Chronic Health Evaluation; ARDS, acute respiratory distress syndrome; DU, duodenal ulcer; F, female; GI, gastrointestinal; ICU, intensive care unit; LOS, length of stay; M, male.

that this had on other ADLs and energy levels. Poor sleeping patterns were reported at all stages of the journey to recovery for patients. One patient described their altered sleeping habits and the impact that this had:

That's all I was doing...sleeping. Then I would get up about two o'clock in the morning maybe for two hours, then I would go back to sleep again. My sleeping pattern was very erratic. Even now, if I don't get a sleep in the afternoon...well I get grumpy.

Discharge planning and communication across different areas within the acute care service was seen as lacking and, in some cases, caused significant stress and upset in participants. As a result, there may be a significant impact on physical and psychological recovery and on daily routine. There were key areas identified by participants: the journey through the hospital, medicines reconciliation, the community interface and access to appropriate housing or suitably adapted homes. These

\section{Table 2 Overview of interviews}

\begin{tabular}{ll}
\hline Gender (\% male) & 71.4 \\
Age (mean, range) & 49 \\
APACHE II (mean, range) & $22.5(9-33)$ \\
Length of interviews in minutes & $41(17-90)$ \\
(mean, range) & \\
ICU LOS (median, IQR) & $6-11.25)$ \\
\hline APACHE, Acute Physiology and Chronic Health Evaluation; ICU \\
LOS, intensive care unit length of stay.
\end{tabular}

issues affected people's ability to carry out different ADLs in a way which was acceptable to them. One participant discussed issues with medicines reconciliation:

\begin{abstract}
Dr **** phoned me up and he said "do you know you weren't getting Metformin and something else when you were in the hospital?" I went, "to be honest with you doctor I haven't a clue what I was getting in hospital. I just took what I was given." He said "well, they took you off Metformin and they took you off, I think it was about five or six tablets."
\end{abstract}

\section{Impact of psychological resilience of recovery}

During the interviews, many participants described a loss of control and self-efficacy in different aspects of their lives. However, some participants also explained how they had regained it; in essence, they discussed the construction of resilience and coping mechanisms for these problems. Individuals gave details of an inner drive' or 'self-determination' and how goal setting, for example with the physiotherapist, had accelerated their recovery.

Unfortunately, many participants had not regained control of their lives at the time of interview. Indeed, some felt that their lives were completely out of control. This issue was particularly stark in the group admitted with a background of AUDs. Many participants in this group felt they still had no control over their lives and this had an influence on their recovery from critical care and their relationship with alcohol. However, part of the reason why many participants continued to drink excessively and perhaps were unable to regain a sense of 
Table 3 Themes and superordinate themes produced for qualitative findings

\begin{tabular}{|c|c|}
\hline Themes & Superordinate themes \\
\hline $\begin{array}{l}\text { Impact on activities of } \\
\text { daily living }\end{array}$ & $\begin{array}{l}\text { Psychological problems } \\
\text { (eg, PTSD, depression, } \\
\text { anxiety) } \\
\text { Physical problems } \\
\text { (eg, poor mobility and } \\
\text { chronic pain) } \\
\text { Discharge planning }\end{array}$ \\
\hline $\begin{array}{l}\text { Impact of psychological } \\
\text { resilience on recovery }\end{array}$ & $\begin{array}{l}\text { Loss of control } \\
\text { Maintenance of } \\
\text { self-efficacy } \\
\text { Ownership of the journey }\end{array}$ \\
\hline $\begin{array}{l}\text { Social support and } \\
\text { cohesion }\end{array}$ & $\begin{array}{l}\text { The role of positive and } \\
\text { negative social support } \\
\text { Social isolation } \\
\text { (participants) } \\
\text { Social isolation and strain } \\
\text { (families and carers) }\end{array}$ \\
\hline $\begin{array}{l}\text { Recovery and support for } \\
\text { alcohol-related } \\
\text { admissions }\end{array}$ & $\begin{array}{l}\text { Interaction with healthcare } \\
\text { professionals } \\
\text { Appropriate and timely } \\
\text { rehabilitation for alcohol } \\
\text { misuse } \\
\text { Impact of critical illness on } \\
\text { alcohol-related behaviours }\end{array}$ \\
\hline
\end{tabular}

control was the challenging health issues, both acute and chronic, which they were facing.

One of the reasons many participants in this study gave for this feeling of 'loss of control' was a lack of ownership over their experience, health and critical care journey. This lack of memory of the ICU stay resulted in some participants finding it difficult to understand how seriously unwell they had been and often meant that individuals struggled to comprehend why fundamental aspects of their life, such as physical functioning, were so difficult. As a result, they found it difficult to fully engage with their recovery.

Patients interviewed discussed how they had received very little information about their time in the ICU to help improve these gaps in memory and the impact that this had:

It would have helped if somebody had actually explained, you're leaving ICU, this is what we have done for you... this is what's gone wrong and we've done this...you know, just to bridge the gap a little bit.

An interesting finding from this work was, if participants with a background of an AUD understood how seriously unwell they had been and had ownership of their story, they were more likely to make positive steps forward in their recovery and in changing their relationship with alcohol:

I'm glad, I'm actually glad that I do remember things that happened to me. Because when I got out of hospital
I've got that in my mind now and see the thought of alcohol and all that, I just think of the way...lying in hospital and that's not going to happen to me anymore.

\section{Social support and cohesion}

Many participants described what they perceived as positive and negative types of social support during recovery from ICU. Those with an AUD spoke of negative social environments and social structures more frequently than those without an AUD.

The presence of effective social support networks helped individuals recover from critical illness. These included close family and carers as well as other forms of support, including employers and friends. These formal and informal support networks were key at all stages of the journey to recovery, including inside the hospital setting. When this infrastructure was not present, it led to a feeling of isolation. Negative social structures included poor housing and access to stable housing as well as family members and friends with similar alcohol and lifestyle problems.

\section{Recovery and support for alcohol-related admissions}

Many participants discussed the importance of appropriate interactions with healthcare professionals during their recovery. A small number of participants stated that they were upset when their alcohol intake was not assessed properly and 'judgements' were made about their alcohol consumption. However, on the whole, participants discussed the positive influence of clear and honest communication about the impact of alcohol on their health:

The consultants (the Attending) have told me and things like that. It's actually been drilled into my head that this is what's going to happen if you do it again.

A major aspect of recovery which was explored with participants was the role of rehabilitation and support for AUDs during recovery from critical illness. There were two key parts to this discussion: the need for appropriate interventions and the timing of these interventions. Many participants discussed their pre-existing relationships with specific alcohol workers in the community. They stated that the rehabilitation offered within the acute care setting did not take these relationships into account. As a result, some participants refused the support offered in the ward setting. Participants also articulated the importance of appropriate timing for addiction worker input. Many could not remember any contact during their time in the acute care setting, which may suggest that the timing of these interventions was not optimal. One participant tried to reflect on his time in the ward environment and potential input from addiction workers:

I'm assuming that they must have, but I don't remember it. I don't doubt it happened, but I don't remember it at all. 
All participants with a background of an AUD stated that admission to ICU had an impact on their relationship with alcohol. During the interviews, many participants spoke about their admission to ICU acting as a 'wake-up call'. For those patients who had stopped drinking completely, many reported positive benefits not just with their health, but in other aspects of their life. For example, participants spoke of the positive impact that sobriety had on relationships with family members and other aspects of their health such as eating and exercising:

My health is a lot better. Not drinking...I'm more active with my wife and kids, getting more involved with them, I'm just trying to build my life back up to a good standard now, compared to what it was previously. I'm more positive, positive life for myself now compared to what I was before.

However, some patients continued to drink harmfully after discharge from ICU. Many reasons were given for this, with social reasons being the most prominent. Other factors included housing and ongoing medical concerns such as chronic pain:

But the reason I go on a binge is because of the pains.... I was almost crying myself to sleep at night. I know that sounds like an excuse....

Online supplementary file 2 provides further quotes related to each theme presented.

\section{DISCUSSION}

This is the first study, as far as we can establish, which has explored recovery in critically ill patients with AUDs beyond the hospital setting. Participants discussed a significant and complex interplay between AUDs and recovery from a physical, social and psychological perspective. Participants also explored the impact of critical illness and the impact that this had on their relationship with alcohol moving forward.

Similar to many previously published studies, participants described ongoing physical, psychological, social and emotional problems during recovery from critical illness. ${ }^{1-5}$ The physical problems which were discussed were consistent with previous literature on recovery from intensive care and included poor mobility and chronic pain issues. ${ }^{2}$ These physical problems influenced many parts of recovery including psychological health and dignity. For many participants with a background with an AUD, it also impacted on their ability to move forward with reducing their alcohol intake. However, the use of goal-directed therapy and setting individualised goals for physical and psychological recovery seemed to be of benefit. The recent study by Walker $e t a t^{23}$ also discussed the benefits of making focused care plans with patients. Future rehabilitation for this cohort should explore supporting patients during recovery with the use of individualised and personal goals. ${ }^{23}$

The emergence of, in some cases, serious psychological issues following intensive care discharge was discussed extensively by the patients in this cohort. Low mood, anxiety, poor memory and concentration and persistent fatigue were some of the commonly encountered issues. The impact of poor sleeping patterns on all aspects of recovery was also apparent for patients and carers. There is an abundance of literature which has described ongoing psychological issues for ICU survivors. ${ }^{424}$ There have been some promising findings emerging from the research regarding ICU diaries to overcome some of these issues. However, more work is required with ICU diaries, especially with regard to format and optimal time frames for dissemination. ${ }^{25} 26$

A further finding of this study was the impact of new pharmacy regimes, or indeed the lack of medicines reconciliation for participants when discharged home from hospital. There were a number of participants who had no understanding of their drugs, and there were a number of significant errors which had occurred. There is some emerging evidence regarding the impact of medication issues post intensive care discharge. ${ }^{27}$ More work is required in this area to ensure potentially life-threatening errors are reduced and appropriate interventions put in place.

Psychological resilience and its impact on recovery also emerged as a theme from the in-depth, semistructured interviews. Patients frequently discussed how they felt they had lost control over their health, and in some cases they felt they had no command over their future. This problem was particularly prevalent in those patients with a background of an AUD. This is consistent with the small body of literature in the field. Connolly $e t a t^{28}$ found that patients with ongoing psychological distress or problems found the maintenance of self-efficacy particularly challenging after critical illness. One of the reasons many participants in this study gave for this feeling was a lack of ownership over their experience, health and critical care journey. This lack of ownership was related to poor memory and the inability to differentiate between delusional memories and reality. There has been a focus on producing the 'patient story' in various forms as part of different approaches to rehabilitation. The findings from this work would suggest that these are helpful interventions. However, the evidence suggests that these approaches in isolation are not enough to support patients and family members through the difficult recovery trajectory which is often encountered. A more holistic model of care, which features a component on rebuilding the patient journey, is needed.

From these interviews, it appeared that participants with an AUD, who positively changed their behaviour with alcohol after ICU, were the only group who could possibly have a constructive and indeed positive change in their well-being after ICU. Another finding from this 
work was that those patients who had clear memories of the ICU stay and how seriously unwell they had been discussed changing their relationship with alcohol more frequently. It may be that interventions such as the ICU diary may be appropriate for this group to give meaning and comprehensibility to their critical illness. This could be an area of future research regarding ICU diaries and AUDs within the critical care environment.

Patients with AUDs also discussed mechanisms for appropriate support for alcohol-related behaviours after discharge from critical care. Similar to Clark et al, ${ }^{17}$ patients discussed the importance of clear, honest and empathic communication and interactions with healthcare professionals. Furthermore, participants discussed the importance of timely and appropriate rehabilitation after critical care for optimal support for their addiction. This includes the delivery of support when a patient is aware of the intervention and at a time which is suitable for the individual. Similar to previous research, this study has demonstrated that an admission to critical care could represent a 'teachable moment'; however, more evidence is required into how and when any intervention should be delivered. ${ }^{29}$

\section{Study limitations}

This study was undertaken in a single centre, in an area of high deprivation where alcohol-related illness is a significant public health issue. Glasgow has high rates of unemployment, with a significant number of individuals out of work because of ill health. ${ }^{30}$ Owing to the singlecentre nature of this study, it can only provide information on the range of experiences described by the participants interviewed. As a result, conclusions about how prevalent such experiences are cannot be made. A further limitation of this work is that those patients who participated in the semistructured interviews were a selfselecting group and may not have been completely representative of the population being explored. Last, most interviews took place within the hospital setting. This may have resulted in a specific cohort of patients not being involved in the study-those who did not wish to or could not attend the hospital environment.

\section{CONCLUSION}

There is a significant interplay between alcohol misuse and recovery from critical illness. This study has demonstrated that at present, there is a haphazard approach to rehabilitation for all patients after ICU. A more targeted rehabilitation pathway for all patients leaving critical care, with specific emphasis on alcohol misuse if appropriate, requires to be generated.

Twitter Follow Joanne McPeake at @jomcpeake22

Acknowledgements The authors would like to thank the participants of the interviews who took the time to take part in this study.

Contributors JMcP collected and analysed data and was the main author. AO and EF acted as research supervisors for the work and contributed to the final presentation of the work. TQ collected data and contributed to the final presentation of the work. JK provided overall supervision of this project and contributed to the presentation of this work.

Funding This work was supported by a TENOVUS Scotland small grant (£5124). Joanne McPeake was the main grant holder. JMcP was also supported by a Research Scholarship from the Florence Nightingale Foundation, London. The funding bodies are both charitable organisations; they had no role in the design, collection, analysis, interpretation of data or in the writing of the manuscript. They had no role in the decision to submit the manuscript for publication. All authors have read and approved the manuscript.

Competing interests None declared.

Ethics approval West of Scotland Research Ethics Committee (Reference Number 12/WS/0039).

Provenance and peer review Not commissioned; externally peer reviewed.

Data sharing statement No additional data are available.

Open Access This is an Open Access article distributed in accordance with the Creative Commons Attribution Non Commercial (CC BY-NC 4.0) license, which permits others to distribute, remix, adapt, build upon this work noncommercially, and license their derivative works on different terms, provided the original work is properly cited and the use is non-commercial. See: http:// creativecommons.org/licenses/by-nc/4.0/

\section{REFERENCES}

1. Quasim T, Brown J, Kinsella J. Employment, social dependency and return to work after intensive care. J Intensive Care Soc 2015;16:31-6.

2. Herridge MS, Tansey CM, Matte A, et al. Functional disability five years after Acute Respiratory Distress Syndrome. N Engl J Med 2011;364:1293-304.

3. Griffiths J, Hatch RA, Bishop J, et al. An exploration of social and economic outcomes and associated health related quality of life after critical illness in general intensive care unit survivors: a 12 month follow up study. Crit Care 2013;17:R100.

4. Wade DM, Howell DC, Weinman JA, et al. Investigating risk factors for psychological morbidity three months after intensive care: a prospective study. Crit Care 2012;16:R192.

5. Battle CE, Lovett $\mathrm{S}$, Hutchings $\mathrm{H}$. Chronic pain in survivors of critical illness: a retrospective analysis of incidence and risk factors. Crit Care 2013;17:R101.

6. Fletcher SN, Kennedy DD, Ghosh IR, et al. Persistent neuromuscular and neurophysiologic abnormalities in long term survivors of prolonged critical illness. Crit Care Med 2003;31:1012-16.

7. Tembo AC, Parker V, Higgins I. The experience of sleep deprivation in intensive care patients: findings from a larger hermeneutic phenomenological study. Intensive Crit Care Nurs 2013;29:310-16.

8. Mostafa SM, Murthy VS. Alcohol related admissions to an adult intensive care: an audit. Eur J Anaesthesiol 2002;19:193-6.

9. Uusaro A, Parviainen I, Tenhunen JJ, et al. The proportion of intensive care unit admissions related to alcohol use: a prospective cohort study. Acta Anaesthesiol Scand 2005;49:1236-40.

10. O'Brien JM, Lu B, Ali NA, et al. Alcohol Dependence is independently associated with sepsis, septic shock and hospital mortality among adult intensive care unit patients. Crit Care Med 2007;35:345-50.

11. McKenny M, O’Beirne S, Fagan C, et al. Alcohol related admission to an intensive care unit in Dublin. Ir J Med Sci 2010;179:405-8.

12. Geary T, O'Brien P, Ramsay S, et al. A national service evaluation of the impact of alcohol on admissions to Scottish Intensive Care Units. Anaesthesia 2012;67:1132-7.

13. Gacouin A, Tadie JM, Uhel F, et al. At risk drinking is independently associated with ICU and one year mortality in critically ill non trauma patients. Crit Care Med 2014;42:860-7.

14. McPeake JM, Shaw M, O'Neill A, et al. Do alcohol use disorders impact on long term outcomes from intensive care? Crit Care 2015;19:185.

15. Christensen S, Johansen MB, Pedersen L, et al. Three year mortality among alcoholic patients after intensive care: a population based cohort study. Crit Care 2012;16:1-26.

16. Gentilello LM. Alcohol and the Intensive Care Unit: it's not just an antiseptic. Crit Care Med 2007;35:627-8. 
17. Clark BJ, Jones J, Cook $P$, et al. Facilitators and Barriers to initiating change in medical intensive care unit survivors with alcohol use disorders: a qualitative study. J Crit Care 2013;28:849-56.

18. Smith JA, Flowers P, Larkin M. Interpretative phenomenological analysis. London: Sage Publications, 2009.

19. Scottish Government. Scottish Index of multiple deprivation. A National Statistics Publication for Scotland. Edinburgh: Scottish Government, 2012.

20. Health Organisation. World Health Organisation Definition of Diseases. 2010. http://www.who.int/substance_abuse/terminology/ ICD10ResearchDiagnosis.pdf?ua=1 (accessed 18 Apr 2012).

21. McPeake JM, Bateson M, O'Neill A, et al. Assessment and management of alcohol related admissions to UK intensive care unit. Nurs Crit Care 2013;18:187-92.

22. Intensive Care Society. Levels of critical care for adult patients. London: The Intensive Care Society, 2009.

23. Walker W, Wright J, Danjoux G, et al. Project Post Intensive Care exercise (PIX): a qualitative exploration of intensive care unit survivors perceptions of quality of life post discharge and experience of exercise rehabilitation. $J$ Intensive Care Soc $2015 ; 16: 37-44$
24. Davydow DS, Gifford JM, Desai SV, et al. Posttraumatic stress disorder in general intensive care unit survivors: a systematic review. Gen Hosp Psychiatry 2008;30:421-34

25. Jones C, Backman CG, Capuzzo M, et al. Intensive Care diaries reduce new onset Post traumatic stress disorder following critical illness: a randomised control trial. Crit Care 2010; 14:168-78.

26. Aitken LM, Rattray J, Hull A, et al. The use of patient diaries in psychological recovery from intensive care. Crit Care 2013;17:253.

27. Eijsbroek H, Howell DCJ, Smith F, et al. Medication issues experienced by patients and carers after discharge from the intensive care unit. J Crit Care 2013;28:46-50.

28. Connolly FR, Aitken LM, Tower M, et al. Factors associated with self efficacy for managing recovery in the trauma intensive care population. A prospective cohort study. Injury 2014;45:272-8.

29. Clark BJ, Moss M. Secondary Prevention in the intensive care unit: does intensive care unit admission represent a teachable moment? Crit Care Med 2011;39:1500-6.

30. Brown J, Hanlon P, Turok I, et al. Mental Health as a reason for claiming incapacity benefit- a comparison of national and local trends. J Public Health (Oxf) 2009;31:74-80. 